\title{
Anticorrelation between Ion Acceleration and Nonlinear Coherent Structures from Laser-Underdense Plasma Interaction
}

\author{
F. Sylla, ${ }^{1}$ A. Flacco,${ }^{1}$ S. Kahaly,${ }^{1}$ M. Veltcheva,${ }^{1,2}$ A. Lifschitz, ${ }^{1}$ G. Sanchez-Arriaga,${ }^{3}$ E. Lefebvre,${ }^{3}$ and V. Malka ${ }^{1}$ \\ ${ }^{1}$ Laboratoire d'Optique Appliquée, ENSTA, CNRS, \\ Ecole Polytechnique, UMR 7639, 91761 Palaiseau, France \\ ${ }^{2}$ Dipartimento di Fisica "G. Occhialini", Università degli Studi di Milano-Biccoca, piazza della Scienza 3, 20126 Milan, Italy \\ ${ }^{3}$ CEA, DAM, DIF, 91297 Arpajon, France
}

(Dated: October 27, 2011)

\begin{abstract}
In laser-plasma experiments, we observed that ion acceleration from the Coulomb explosion of the plasma channel bored by the laser, is prevented when multiple plasma instabilities such as filamentation and hosing, and nonlinear coherent structures (vortices/post-solitons) appear in the wake of an ultrashort laser pulse. The tailoring of the longitudinal plasma density ramp allows us to control the onset of these insabilities. We deduced that the laser pulse is depleted into these structures in our conditions, when a plasma at about $10 \%$ of the critical density exhibits a gradient on the order of $250 \mu \mathrm{m}$ (gaussian fit), thus hindering the acceleration. A promising experimental setup with a long pulse is demonstrated enabling the excitation of an isolated coherent structure for polarimetric measurements and, in further perspectives, parametric studies of ion plasma acceleration efficiency.
\end{abstract}

Laser-underdense plasma acceleration rests on the interaction of an intense relativistic pulse $\left(I \lambda^{2}>10^{18}\right.$ $\mathrm{Wcm}^{-2} \mu \mathrm{m}^{2}$ ) with a plasma in a large range of electronic density $\left(n_{e} \in\left[10^{18}, 10^{20}\right] \mathrm{cm}^{-3}\right)$ [1-4]. In particular, the Coulomb explosion of a positively charged channel, created by the laser as it propagates in the plasma, is an efficient way to radially accelerate ions [1, 5]. However, this acceleration can be accompanied by the copious generation of non-linear plasma structures that significantly deplete the pulse energy, theoretically up to $30-40 \%$ of the laser energy [6] 10. These structures aroused the interest of numerous theoretical and experimental studies [6, 8, 11 15]. An underdense plasma with $n_{e} \sim$ tens of percent of $n_{c}, n_{c}$ being the critical density, is particularly suitable for the excitation of coherent structures like electromagnetic solitons and electron vortices, as predicted in Refs. [6, 8, 10, 11] and observed with proton radiography diagnostics in Refs. [12, 13, 16. In Ref. 6, a $3 \mathrm{D}$ electromagnetic cavity structure in the wake of the pulse, termed transverse magnetic-soliton, is identified and slowly expands into a post-soliton, as ions within the cavity explode to energies of tens of $\mathrm{keV}$. This example illustrated a novel ion acceleration scheme from underdense plasma, different from the channel Coulomb explosion 1, Target Normal Sheath Acceleration-like process [3], or longitudinal magnetic vortex acceleration [17].

In this letter, we present for the first time an "inverse correlation" (mutual exclusion) between the ion acceleration by radial Coulomb explosion and the generation of nonlinear coherent structures in the wake of the laser pulse. Our results tend to prove that the efficiency of the laser ion acceleration directly from a gas jet is highly dependent upon the density gradient profile. In our case, though the density ramp should contribute to the increase of the laser intensity by self-focusing of the pulse [18, 19, it induces filamentation [19, hosing instabili- ties 20] and ultimately generation of nonlinear coherent structures, the overall turning out to be detrimental for the acceleration.

The experiment was conducted at the Laboratoire d'Optique Appliquée with the Ti:Sapphire laser "Salle Jaune", which delivers an ultrashort (duration $\tau=30$ fs), linearly-polarized (along $z$, see FIG. 1) pulse with $E_{L} \sim 810 \mathrm{~mJ}$ on target [21. The pulse was focused with an $f / 10$ off-axis parabolic mirror to a $25-\mu \mathrm{m}\left(1 / e^{2}\right)$ focal spot onto a $700 \mu \mathrm{m}(\mathrm{FWHM})$ supersonic He jet at $\sim 300 \mu \mathrm{m}$ above the nozzle exit. The vacuum laser intensity is $I_{0} \sim 1 \times 10^{19} \mathrm{Wcm}^{-2}$. With $n_{e}=1-10 \times 10^{19}$ $\mathrm{cm}^{-3}$, it gives a ratio $P_{L} / P_{c}=5.3-53$ (with $P_{L}$ the laser power and $P_{c}=16.2 n_{c} / n_{e} \mathrm{GW}$ the critical power for self-focusing). Before focusing, the beam was split and $10 \%$ of the energy sent onto a BBO crystal for second harmonic generation. The intensity and spatial quality of this collimated frequency-doubled beam were improved by passing through a $50-\mu \mathrm{m}$-pinhole-filtered beam reducer. It was finally incident on the jet normally to the main laser axis for illuminating and probing the interaction transversally (see FIG. 1). Measurements of ion emission and plasma probing (interferometry and polarimetry), both at $90^{\circ}$ from the main laser propagation axis, were simultaneously done by means of a $45^{\circ}$-pierced mirror reflecting the probe beam onto the gas jet without cutting the ion beam (see FIG. 1).

The ion detector is composed of a $100-\mu \mathrm{m}$ spatial filter (solid angle acceptance is $1.2 \times 10^{-8} \mathrm{sr}$ ) with a Thomson parabola spectrometer coupled to 40-mm imaging microchannel plate chevron assembly (see FIG. 1(a)). A linearized 16-bit CCD camera $(1024 \times 1024$ pixels $)$ enables single-shot recordings of scintillating traces and thus shot-to-shot statistical analyses of ion spectra.

An $f / 1$ achromatic doublet coupled with a 50/50 beam splitter enables to record at the same time with 


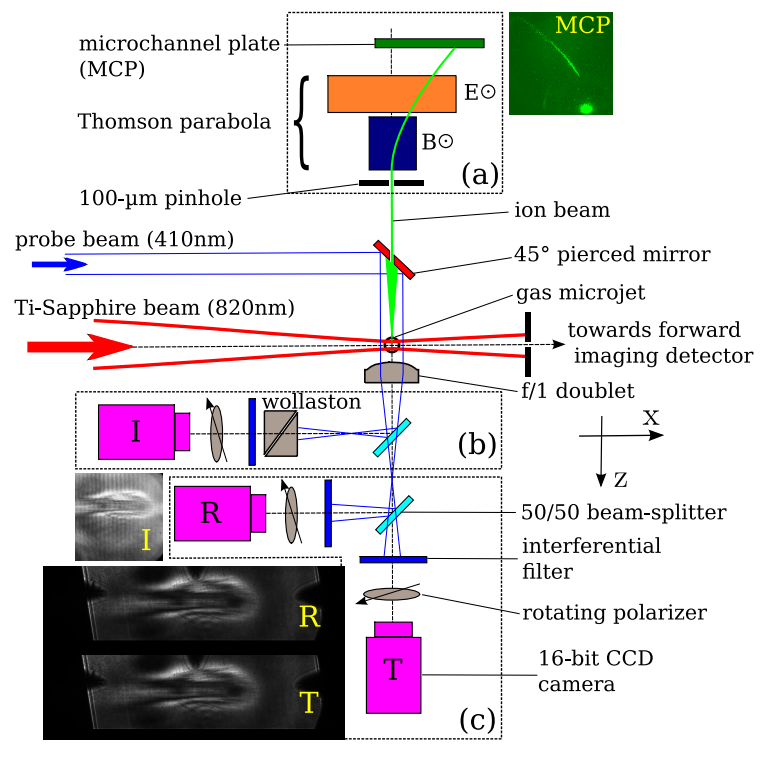

FIG. 1: Experimental setup for the interaction of a microjet and a short Ti-Sapphire pulse, illuminated by a linearlypolarized-frequency-doubled probe pulse. (a) Imaging Ion spectrometer along the normal to the main beam with Thomson parabola $(B=0.3 \mathrm{~T})$ and chevron microchannel plate (MCP). A second ion spectrometer is also set on the laser axis. (b) Nomarski interferometer. (c) Polarimeter using Faraday rotation effect. For Polarigrams $\mathrm{R}$ and $\mathrm{T}$, the polarizers are rotated by respectively +10 and $-10^{\circ}$ fom the extinction

spatial resolution $\sim 1 \mu \mathrm{m}$, interferograms encoding the plasma density variations using a Nomarski interferometer [22] (see FIG. 11(b)), and polarigrams revealing the component of the magnetic field collinear to the probe beam direction (see FIG. 1(c)). The interferometer contains a Wollaston biprism of $10 \times 10 \mathrm{~mm}^{2}$ with a 410 $\mathrm{nm}$ interferential filter to improve the temporal coherence of the interferences. The polarimeter is composed of two identical assemblies, each comprising in particular a Glan Laser polarizer (extinction ratio better than $10^{-4}$ ) and a 16-bit CCD camera. For each assembly, a scan of the rotating polarizer was carried out for probe beam transmission calibration (every five degrees over the entire rotation). For the measurements, the polarizers were detuned by a common angle from each minimum of transmission (typical detunings $\theta_{R}=+10$ and $\theta_{T}=-10^{\circ}$ for respectively the transmitted and reflected images from the beam splitter, see FIG. 11(c)).

The magnetic field detection rests on the measurment of the rotation, due to Faraday effect, of the incident linear polarization of the probe beam electric field when it crosses the plasma [23, 24. The total rotation angle along a path $l_{z}$ is given by $\phi_{\text {rot }}(x, y)=$ $\frac{e}{2 m_{e} c n_{c}} \int_{l_{z}} n_{e}(x, y, z) B_{z} d z$. Here $B_{z}$ is the magnetic field component collinear to the probe beam direction, $n_{c}$ the plasma critical density for the probe beam, $e$ and $m_{e}$ the mass and charge of the electron and $c$ the light velocity in vacuum. Taking the intensity ratio from the

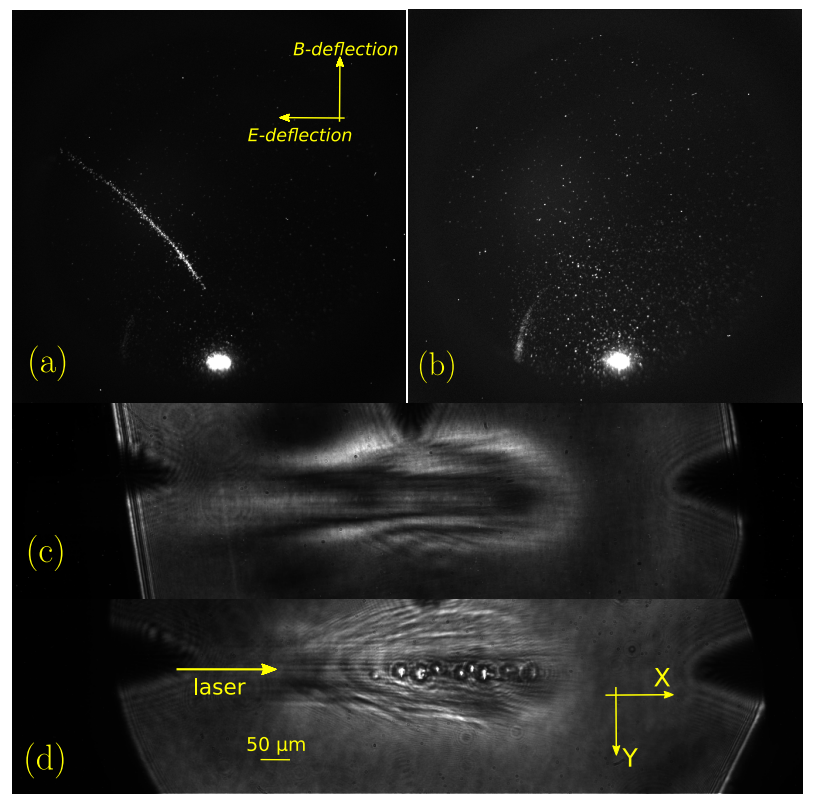

FIG. 2: (a) and (b): MCP recordings normally to the laser axis for $n_{e} \sim 10^{20} \mathrm{~cm}^{-3}$. (a) short plasma gradient $\sim 150 \mu \mathrm{m}$. $\mathrm{He}^{+}$trace with energy cutoff $\sim 250 \mathrm{keV}$. (b) long plasma gradient $\sim 250 \mu \mathrm{m}$. (c) and (d): Shadowgram at 1 ps after the pulse enters the jet (resolution $\sim 1 \mu \mathrm{m}$, magnification 8.2). (c) short plasma gradient. (d) long plasma gradient. The shadowgram shows a clear train of bubble-like structures in the laser wake. The laser polarization is normal to the picture (S-polarized)

two shadowgrams $\mathrm{R}$ and $\mathrm{T}$ obtained with opposite detuning angles (see FIG. 1(c)) prevents the extracted $\phi_{\text {rot }}$ map from being corrupted by probe beam shot-to-shot fluctuations in intensity, and image shift from plasma refraction or vibrations. However, this requires a pixelto-pixel correspondance of the pictures. We achieved a sub-pixel-accurate matching of the two pictures by tagging the field of view at three reference-areas prior to the beam-splitting, and by running a numerical pattern matching routine.

Figure 2 shows the MCP recordings at $90^{\circ}$ from the laser axis for two plasma gradient conditions. In (a), the laser is shot on the gas jet at an early stage of its expansion in the vacuum chamber so that the plasma gradient is steep $\sim 150 \mu \mathrm{m}$ (with a gaussian fit). In (b), the interaction occurs at a late time of the expansion $(80 \mathrm{~ms}$ after the closing of the valve), and the gradient is smooth $\sim 250 \mu \mathrm{m}$. For both conditions, the peak density in the jet has the same value $n_{e} \sim 10^{20} \mathrm{~cm}^{-3}$. We observe systematically radial ion acceleration from Coulomb explosion in case (a) and no trace in (b). In both cases, no ion was detected in the forward direction (see FIG. 1 for the geometry). When acceleration occurs, only $\mathrm{He}^{+}$are detected from $12 \mathrm{keV}$ to cutoff energies of $250 \mathrm{keV}$. We attribute the absence of $\mathrm{He}^{2+}$, though the laser peak intensity is 1000 times higher than the helium potential of full ionization, to efficient recombination of this species 
in the neutral corona starting at $100 \mu \mathrm{m}$ from the laser axis. Indeed, the electron capture is the most probable channel of interaction (cross section $\sigma \sim 10^{16} \mathrm{~cm}^{-2}$ ) for $\mathrm{He}^{2+}$ projectile of $250 \mathrm{keV}$ streaming in He gas 25. In panel (d), one can see the typical shadowgram (revealing sharp electron density fronts) obtained in case (b). A train of zig-zag ordered and well separated bubble structures with an average size of about $30 \mu \mathrm{m}\left(\gg \lambda_{p}=c / \omega_{p e}\right.$, the plasma wavelength) can be clearly seen in the wake of the laser pulse. The bubbles have a bright spot in their centers that is not observable when the probe beam is turned off. This means that the emission is not of selfemission nature and results from refractive effects of the probe light by these structures behaving like microlenses. An interesting point is the quasi-circular shape of these structures, meaning that, during the transverse illumination of $30 \mathrm{fs}$, their centers are quasi-static in the picture plane. In the short-plasma scale length case, for which transverse ion acceleration is detected (Fig. 2(a)), no such isolated structure is ever observed in the gas jet (Fig. 2(c)).

To understand this inverse correlation, we investigated in time the interaction in the long gradient case. Figure 3 presents a sequence of five shadowgrams taken at different delays after the pulse enters the jet $\left(t_{0}\right)$. Very early in the interaction $\left(t_{0}+540 \mathrm{fs}\right)$, sizeable filaments develop next to the main laser channel [19] (see arrows in panel (b) from Fig. 3). As the pulse propagates along, the filamentation becomes stronger and multiple circular structures appear about the laser channel, near the center of the jet. In this time-resolved scan, we observe that the structures appear randomly in the laser path and no correspondance could be drawn between the pulse and bubble positions. Moreover, even if this shadowgram diagnostic is not intrinsically time-resolved, contrary to the proton radiography technique [26], the stability of the interaction was sufficient to assess statistically the average size of the structure until the moment when the shadowgrams become blurred and unexploitable, at about 7 ps after the onset of the interaction. Over that timespan, we observed that the structures have fairly the same size, irrespective of their positions to the laser front and measured an average increase of less than $10 \mu \mathrm{m}$ in size.

Besides being copiously generated by the interaction, the nonlinear structures distribute spatially into two remarkable patterns, identifiable by following the bright central blobs, which arose randomly in our experiment. Figure 4 illustrates both antisymetric (zig-zag about the laser axis) (panel (a)) and axial (panel (b)) distributions of the bubbles. In Ref. 27, the authors carried out 2D particle-in-cell simulation with $a_{0}=3, \tau=30 \mathrm{fs}$ and about $1 \mathrm{~mm}$ of plasma at $n_{e}=10^{19} \mathrm{~cm}^{-3}$. They obtained for initially $\mathrm{p}$ - and s-polarized light (i.e. an electric field collinear and normal to the observation plane), respectively antisymetric and axial density map patterns resembling our experimental results (see figures 3 and

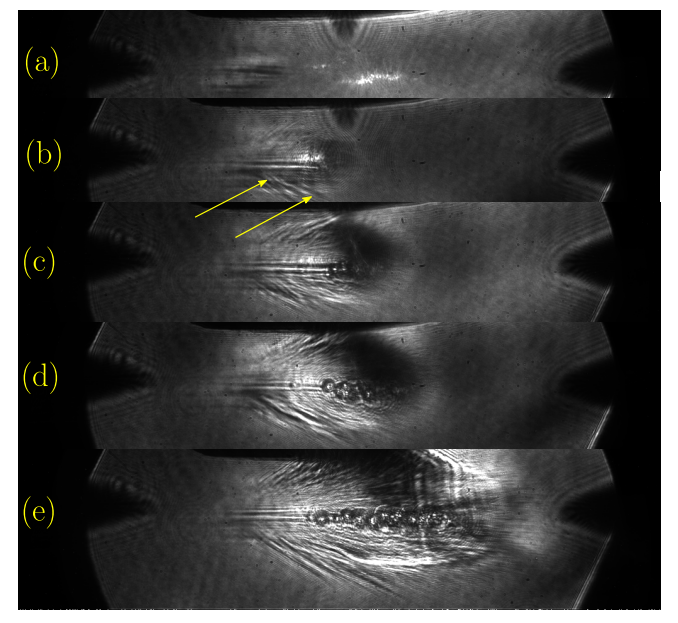

FIG. 3: Time-resolved pulse penetration in the plasma gradient. (a) reference instant $t_{0}$, (b) $t_{0}+540 \mathrm{fs}$, (c) $t_{0}+940 \mathrm{fs}$, (d) $t_{0}+1.54 \mathrm{ps}$, (e) $t_{0}+2.84 \mathrm{ps}$. Arrows indicate the growth of prominent laser filaments.

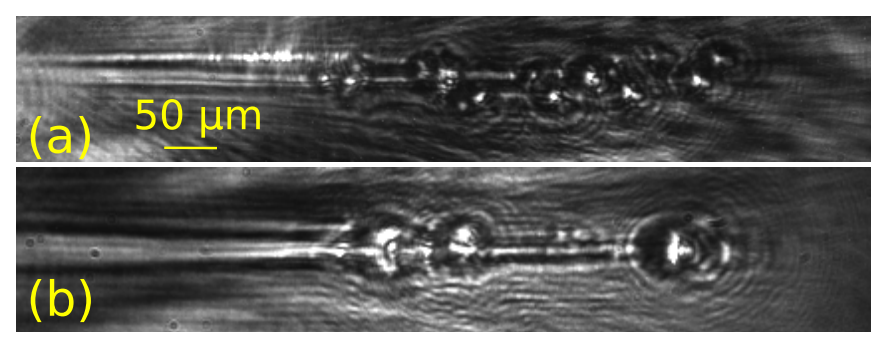

FIG. 4: Spatial distribution of the bubbles for two shots in the same conditions at $t_{0}+1.54 \mathrm{ps}$ showing (a) antisymetric (zigzag) and (b) symetric axial patterns about the main plasma channel

4 in [27]). In particular, the antisymetric modulation is ascribed to the hosing instability (see also 9]). Although in our experiment the incident laser polarization is normal to the observation plane, corresponding to the s-polarization of simulations [9, 27, hosing instabilities have also been observed experimentally in similar conditions [28]. Therefore, following [9, 27, 28], we propose to ascribe our observations to the laser excitation of the hosing instability inducing the developpement of von Karmàn row of electron vortices (a) and to shadowgraphic traces left by post-solitons (b).

At that point, it is clear that the interaction in this operating regime $\left(n_{e} \sim 10^{20} \mathrm{~cm}^{-3}\right.$, long gradient $\sim 250 \mu \mathrm{m}$ ) produces numerous instabilities (filamentation, hosing, vortex/soliton), known to seriously deplete the pulse energy [6, 8, 9, and prevent efficient plasma channel charging for subsequent Coulomb explosion. Moreover, despite their expansions by ion core explosion [9, 12, 15, the bubbles do not provide a detectable acceleration, possibly because of a number of accelerated ions below the detector noise level. Therefore, we were unable to verify the post-soliton acceleration scenario predicted in 6, 15. 
To confirm the electromagnetic nature of the coherent structures, we carried out some polametric measurements. To simplify these measurements, we have identified a regime where few structures are generated at each laser shot, near the center of the jet (statistically close to one). For that, we used a stretched laser pulse $\tau=250 \mathrm{fs}$ carrying the same energy $\left(a_{0}=0.8\right)$ and a small sonic nozzle of $200 \mu \mathrm{m}$ diameter delivering a jet of peak density of few $10^{19} \mathrm{~cm}^{-3}$ which we let expand in vacuum to increase the gradient before the interaction. This setup enables $(i)$ a well-localized interaction, $(i i)$ smoother interferograms and polarigrams without filaments and bubble overlapping ( iii) efficient energy transfer to the structures 9 .

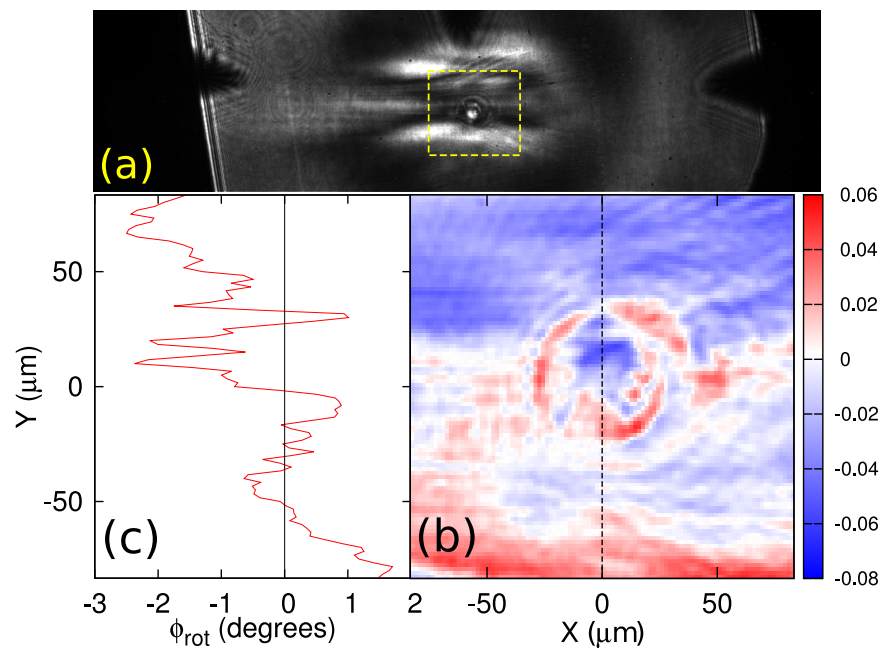

FIG. 5: (a) Shadowgram with one isolated structure in the laser wake at $t_{0}+940 \mathrm{fs}\left(\tau=350 \mathrm{fs}, 10^{19} \mathrm{~cm}^{-3}\right)$. (b) $\phi_{\text {rot }}$ map (in radians) of the yellow square dotted zone in (a). Detuning angle of the polarisers: $\pm 10^{\circ}$. (c) Line-out along the black dotted line in (b)

Figure 5(a) shows a shadowgram with one single spherical structure of $57 \mu \mathrm{m}$ diameter in a clean area. In that simplified case, we were able to extract a $\phi_{\text {rot }}$ map around the structure (panel (b)). From panel (b) and (c), we can deduce that the structure in this condition has an almost axisymmetric polarimetric imprint about the laser polarization direction. The density in the bubble shell is found to be $\sim 7 \times 10^{19} \mathrm{~cm}^{-3}$, five times that of the surrounding plasma. Though the polarimetric information is the convolution of $B_{z}$ by the density along the integrated probe beam path in the plasma, we will assume for the remaining that main contribution comes from the structure and not from the surrounding plasma, as the major part of the bubble seems to be lying in a weakly magnetized area (white region). Doing so, we thus calculate the maximum magnetic field extractable from our data. For $n_{e}=7 \times 10^{19} \mathrm{~cm}^{-3}$, an average path $l_{z}$ in the shell of $25 \mu \mathrm{m}$ (from panel (c)), $n_{c}=4 \times 1.67 \times 10^{21}$ $\mathrm{cm}^{-3}$ and a Faraday rotation of 0.04 radians, we find $B_{z} \leq \frac{\phi_{\text {rot }}}{\frac{n_{e} l e}{2 m_{e} c n_{c}}}=570 \mathrm{~T}$. Though lower, this value is of the same order of magnitude as in the numerical computations in similar conditions [6, 8, and is consistent with the fact that the fields dissipate as the structure expands. The extraction of the space-resolved magnetic field map and a refined magnetic description of the nonlinear structures will be the subject of another publication.

In conclusion, our reproducible observations of bubbles identified as nonlinear coherent structures (vortex/post-soliton) made it possible to demonstrate their detrimental effect on the efficiency of laser-ion accelaration. These structures appear abundantly in the laser wake when the plasma has a long gradient profile in which the laser self-focuses, filaments and develop hosing. When using a small nozzle with lower density, simple conditions with one structure per laser shot can be statistically obtained. We think this all-optical setup represents a powerful method that opens up new opportunity of in-depth investigation of fundamental nonlinear plasma instabilities and also particle acceleration for users of well-spread TeraWatt-class lasers.

The authors thank Prof. S. V. Bulanov for fruitful and encouraging discussions. We are also pleased to acknowledge the support of the European Research Council for funding the PARIS ERC project (contract 226424), and the ANR-08-NT08-1-38025-1. Finally, the authors acknowledge the support of ANR under the GOSPEL project, grant reference ANR-08-BLAN-0072-03.

[1] K. Krushelnick et al., Phys. Rev. Lett. 83, 737 (1999).

[2] V. Malka et al., Nature Physics 44, 447 (2008).

[3] L. Willingale et al., Phys. Rev. Lett. 96, 245002 (2006).

[4] M. S. Wei et al., Phys. Rev. Lett. 93, 155003 (2004).

[5] G. S. Sarkisov et al., Phys. Rev. E 59, 7042 (1999).

[6] T. Esirkepov et al., Phys. Rev. Lett. 89, 275002 (2002).

[7] T. Z. Esirkepov et al., Phys. Rev. Lett. 101, 265001 (2008).

[8] S. V. Bulanov et al., Phys. Rev. Lett. 82, 3440 (1999).

[9] N. M. Naumova et al., Physics of Plasmas 8, 4149 (2001).

[10] Y. Sentoku et al., Phys. Rev. Lett. 83, 3434 (1999).

[11] S. V. Bulanov et al., Phys. Rev. Lett. 76, 3562 (1996).

[12] M. Borghesi et al., Phys. Rev. Lett. 88, 135002 (2002).

[13] L. Romagnani et al., Phys. Rev. Lett. 105, 175002 (2010).

[14] G. Sánchez-Arriaga and E. Lefebvre, Phys. Rev. E 84, 036403 (2011).

[15] G. Sánchez-Arriaga and E. Lefebvre, Phys. Rev. E 84, 036404 (2011).

[16] G. Sarri et al., Phys. Rev. Lett. 105, 175007 (2010).

[17] A. Kuznetsov et al., Plasma Physics Reports 27, 211 (2001).

[18] G. Sun et al., Phys. Fluids 30, 526 (1987).

[19] W. B. Mori et al., Phys. Rev. Lett. 60, 1298 (1988).

[20] P. Sprangle et al., Phys. Rev. Lett. 73, 3544 (1994).

[21] A. Flacco et al., Nuclear Instruments and Methods in Physics Research Section A: Accelerators, Spectrometers, Detectors and Associated Equipment 620, 18 (2010).

[22] R. Benattar et al., Review of Scientific Instruments 50, 
$1583(1979)$.

[23] J. A. Stamper and B. H. Ripin, Phys. Rev. Lett. 34, 138 (1975).

[24] M. C. Kaluza et al., Phys. Rev. Lett. 105, 115002 (2010).

[25] M. B. Shah and H. B. Gilbody, Journal of Physics B:
Atomic and Molecular Physics 18, 899 (1985).

[26] L. Romagnani et al., Phys. Rev. Lett. 95, 195001 (2005).

[27] L. M. Chen et al., Physics of Plasmas 14, 040703 (2007).

[28] M. C. Kaluza et al., Phys. Rev. Lett. 105, 095003 (2010). 